\title{
Differential survival trends of stage II colorectal cancer patients relate to promoter methylation status of $P C D H 10$, SPARC, and UCHL1
}

Ellen Heitzer ${ }^{1}$, Monika Artl ${ }^{1}$, Martin Filipits ${ }^{2}$, Margit Resel ${ }^{3}$, Ricarda Graf ${ }^{1}$, Bettina Weißenbacher ${ }^{3}$, Sigurd Lax ${ }^{4}$, Michael Gnant ${ }^{5}$, Fritz Wrba ${ }^{6}$, Richard Greil ${ }^{7}$, Otto Dietze $^{8}$, Friedrich Hofbauer ${ }^{9}$, Gerhard Böhm ${ }^{10}$, Gerald Höfler ${ }^{11}$, Hellmut Samonigg ${ }^{3}$, Renate Schaberl-Moser ${ }^{3}$, Marija Balic ${ }^{3}$ and Nadia Dandachi ${ }^{3}$

${ }^{1}$ Institute of Human Genetics, Medical University of Graz, Graz, Austria; ${ }^{2}$ Institute of Cancer Research, Department of Medicine I, Comprehensive Cancer Center, Medical University Vienna, Vienna, Austria; ${ }^{3}$ Department of Internal Medicine, Division of Oncology, Medical University of Graz, Graz, Austria; ${ }^{4}$ Institute of Pathology, General Hospital Graz West, Graz, Austria; ${ }^{5}$ Department of Surgery, Comprehensive Cancer Center, Vienna, Austria; ${ }^{6}$ Department of Pathology, Comprehensive Cancer Center, Vienna, Austria; ${ }^{7}$ Third Medical Department, Paracelsus Private Medical University, Salzburg, Austria; ${ }^{8}$ Department of Pathology, Paracelsus Private Medical University, Salzburg, Austria; ${ }^{9}$ Department of Surgery, Hospital Oberpullendorf, Oberpullendorf, Austria; ${ }^{10}$ Department of Pathology, Hospital Oberwart, Oberwart, Austria and ${ }^{11}$ Institute of Pathology, Medical University of Graz, Graz, Austria

Surgical excision of colorectal cancer at early clinical stages is highly effective, but $\mathbf{2 0 - 3 0 \%}$ of patients relapse. Therefore, it is of clinical relevance to identify patients at high risk for recurrence, who would benefit from adjuvant chemotherapy. The objective of this study was to identify prognostic and/or predictive methylation markers in stage II colorectal cancer patients. Therefore, we selected six gene promoters (FZD9, PCDH10 (protocadherin 10), SFRP2, SPARC (secreted protein acidic and rich in cysteine), UCHL1 (ubiquitin carboxyl-terminal hydrolase 1), and WIF1) for methylation analysis in formalin-fixed, paraffin-embedded primary tumor samples of colorectal cancer patients $(n=143)$ who were enrolled in a prospective randomized phase III trial of the Austrian Breast and Colorectal cancer Study Group. Patients were randomized to adjuvant chemotherapy with 5-fluorouracil and leucovorin or surveillance only. Survival analyses revealed that combined evaluation of three promoters (PCDH10, $S P A R C$, and UCHL1) showed differential effects with regard to disease-free survival and overall survival in the two treatment groups (significance level 0.007 ). In the chemotherapy arm, a statistically insignificant trend for patients without methylation toward longer survival was observed $(\boldsymbol{P}=\mathbf{0 . 0 6 9}$ for disease-free survival and $\boldsymbol{P}=0.139$ for overall survival). Contrary, patients in the surveillance arm without methylation in their gene promoters had shorter disease-free survival and overall survival $(P=0.031$ for disease-free survival and $P=0.003$ for overall survival), indicating a prognostic effect of methylation in this group (test for interaction, $P=\mathbf{0 . 0 0 6}$ for disease-free survival and $\boldsymbol{P}=\mathbf{0 . 0 1 8}$ for overall survival). These results indicate that promoter methylation status of $P C D H 10$, SPARC, and UCHL1 may be used both as prognostic and predictive molecular marker for colorectal cancer patients and, therefore, may facilitate treatment decisions for stage II colorectal cancer.

Modern Pathology (2014) 27, 906-915; doi:10.1038/modpathol.2013.204; published online 6 December 2013

Keywords: chemotherapy; colorectal cancer; 5-fluorouracil; methylation; predictive; prognostic; stage II

Correspondence: Dr E Heitzer, PhD, Institute of Human Genetics, Medical University Graz, Harrachgasse 21, 8010 Graz, Austria or M Balic, MD, PhD, Department of Internal Medicine, Division of Oncology, Medical University of Graz, Auenbruggerplatz 15, 8036 Graz, Austria.

E-mail: ellen.heitzer@medunigraz.at or marija.balic@medunigraz.at Received 29 May 2013; accepted 2 September 2013; published online 6 December 2013
Colorectal cancer is one of the most common forms of cancer causing 677000 deaths worldwide per year (http://www.who.int/, July 2008). Although incidence is rising, the death rate from colorectal cancer has been going down for the past 15 years because of improved treatment and screening for early detection. ${ }^{1}$ The tumor is initially slow growing, 
whereas it later often grows rapidly with the development of metastasis in regional and distal lymph node and other sites, including liver and lung. ${ }^{2}$ For patients with stage III colorectal cancer, adjuvant chemotherapy after surgical resection is currently recommended as a standard therapy as it has been shown to improve diseasefree survival and overall survival. ${ }^{3}$ Significant controversy exists regarding the administering of chemotherapy to stage II patients. ${ }^{4}$ Surgical excision at early clinical stages is effective, although in about $20-30 \%$ of patients the disease can recur. Therefore, determining whether a patient with stage II colorectal cancer should receive adjuvant therapy is a challenging decision due to the relative low probability of progression, and the fact that about two-thirds of patients would not benefit from chemotherapy and the costs would be tremendous.

A variety of genetic changes has been identified for colorectal cancer ${ }^{5}$ and some of them have been described as potentially useful biomarkers helping to better identify high-risk patients who would most likely benefit from adjuvant chemotherapy. These markers include, that is, microsatellite instability, ${ }^{6,7}$ loss of heterozygosity of 17p and 18q, expression of thymidylate synthase, ${ }^{8}$ or different gene expression signatures. ${ }^{9-11}$ In colorectal cancer, the commonly found KRAS mutations are considered as negative predictive marker for the use of anti-EGFR therapies; however, prognostic significance of KRAS mutations remains unclear. ${ }^{12}$

In addition to genetic changes, aberrant DNA methylation represents a hallmark of cancer and has been extensively studied in colorectal cancers. ${ }^{13-17}$ Epigenetic signatures have been also shown to serve as potential diagnostic, prognostic, and predictive biomarkers (for a detailed review see Lao and Grady $^{18}$ and Ogino et $a l^{19}$ ). A recurrent methylation pattern referred to as $\mathrm{CpG}$ island hypermethylation was initially observed by Toyota et al. ${ }^{20}$ CpG island hypermethylation has gained a broad acceptance, and although there have been conflicting results about the prognostic or predictive role of $\mathrm{CpG}$ island hypermethylation, ${ }^{21,22}$ Ogino et $a l^{7}$ demonstrated that $\mathrm{CpG}$ island hypermethylation-high status is an independent predictor of cancer survival and seems to eliminate the adverse effect of $B R A F$ mutation, which is associated with a high mortality. However, several studies identified a prognostic role for promoter methylation of genes not included in the $\mathrm{CpG}$ island hypermethylation panel such as MGMT, RASSF1A, or BAGE. ${ }^{23-25}$ Nevertheless, none of these markers has yet found its clinical application.

The aim of our study was to identify new prognostic and/or predictive markers in patients with stage II colorectal cancer who were enrolled in a prospective randomized phase III trial of the Austrian Breast and Colorectal cancer Study Group (Trial 91). ${ }^{26}$

\section{Materials and methods}

\section{Methyl-Profiler}

We analyzed the colorectal cancer cell lines SW480 and Caco-2 using two Methyl-Profiler DNA Methylation PCR Arrays (SA Biosciences, Qiagen, Hilden, Germany). The Human Colon Cancer Methyl-Profiler DNA Methylation PCR Array profiles the methylation status of 24 tumor suppressor genes frequently methylated in colon tumors. The Human WNT Signaling Pathway Methyl-Profiler PCR Array analyzes the promoter methylation status of a panel of 24 promoters of genes involved in WNT signaling during carcinogenesis and cellular differentiation. The assays were performed according to the manufacturer's instructions (for details see Supplementary Material).

\section{Treatment of Colon Cancer Cell Lines with 5-Aza-2- Deoxycytidine}

Colorectal cancer cells Caco-2, HCT116, HT29, and SW480 were obtained from American Type Culture Collection (ATCC, Manassas, VA, USA) and cultured according to their recommendations. Cell lines were authenticated by DNA short-tandem repeat analysis by the Cell Culture Facility of the Center for Medical Research, Medical University Graz (Graz, Austria). To induce demethylation, cells were seeded at a density of $3-6 \times 10^{5}$ cells and allowed to attach in the medium for $24 \mathrm{~h}$. 5-Aza-2deoxycytidine (5-Aza) (Sigma-Aldrich, St Louis, MO, USA) was added to a final concentration of $1 \mu \mathrm{M}$. Cells were incubated for $72 \mathrm{~h}$ with 5 -Aza and the medium was renewed daily. Cells incubated under same conditions without 5-Aza treatment served as a control. After $72 \mathrm{~h}$, cells were harvested and DNA and RNA were extracted for analysis. The experiment was performed in triplicates.

\section{DNA Extraction from Cultured Colon Cancer Cell Lines}

Cell number was measured with CASY (Roche, Penzberg, Germany). DNA was extracted from $5 \times 10^{6}$ or $1 \times 10^{7}$ cells using the Gentra Puregene Kit (Qiagen) according to the manufacturer's instruction for cultured cells.

\section{RNA Extraction from Cultured Colon Cancer Cell Lines}

RNA was extracted using TRIzol Reagent (Invitrogen, Carlsbad, CA, USA) according to the manufacturer's recommendations. RNA was dissolved in 20-50 $\mu$ l RNase free water and stored at $-70^{\circ} \mathrm{C}$.

\section{Quantitative PCR}

A total of 16 genes were selected from MethylProfiler assays for expression analysis in the cell 
lines Caco-2, HCT116, HT29, and SW480 with and without 5-Aza treatment. cDNA from $2 \mu \mathrm{g}$ extracted RNA of three replicates of each cell line was synthesized using Omniscript RT Kit (Qiagen). Expression analysis was performed by quantitative PCR using 25 or $50 \mathrm{ng}$ of cDNA as a template and Fast SYBR ${ }^{\circledR}$ Green Master Mix (Applied Biosystems, Foster City, CA, USA). Each sample was run in technical triplicates. RQ (relative quantification) or fold change was normalized to the lactate dehydrogenase A housekeeping gene using the 2- $\Delta \Delta \mathrm{Ct}$ method. Genes showing an RQ of $>10$-fold in at least one cell line after demethylation were selected and analyzed in patient samples. Primers were designed using Primer3Web 3.0.0 software. Sequences are listed in Supplementary Table 1.

\section{Patients and Tissue Samples}

All tumor specimens were obtained at the time of surgery before adjuvant therapy. Paraffin blocks were stored at room temperature and were identified only by an identification number. A hematoxylin/ eosin-stained section of each tumor block was prepared and used for pathologic confirmation of present carcinoma.

We analyzed tumor samples from a total of 147 patients with stage II colorectal cancer according to the UICC (T3-T4, N0, M0) that were available from Austrian Breast and Colorectal cancer Study Group (Trial 91). ${ }^{26}$ All patients underwent a potentially curative resection without gross or microscopic evidence of residual disease and were recruited for a prospective randomized phase III trial of adjuvant chemotherapy with 5-fluorouracil and leucovorin. Patients were randomized to one of two postoperative treatment arms: (1) 5-fluorouracil/ leucovorin and (2) surveillance only. Chemotherapy was administered as described previously. ${ }^{26}$

\section{DNA Extraction of Formalin-Fixed, Paraffin-Embedded Tissues}

DNA from formalin-fixed, paraffin-embedded tissues was extracted as published previously. ${ }^{27}$ Briefly, tissue samples were sliced into 5- $\mu \mathrm{m}$-thick sections and manually dissected by a pathologist (SL) to reduce the proportion of non-tumor cells in the samples and scraped into an eppendorf tube. The minimum percentage of tumor cells was $40 \%$. Slides were deparaffinized and tissues were lysed with lysis buffer (4M urea, $200 \mathrm{mM}$ Tris, $20 \mathrm{mM} \mathrm{NaCl}, 200 \mathrm{mM}$ EDTA (pH 7.4)) containing proteinase K $(20 \mathrm{mg} / \mathrm{ml}$; Qiagen). DNA was precipitated with isopropanol at $-20^{\circ} \mathrm{C}$ overnight. The DNA pellet was washed, air dried, and dissolved in $20 \mu \mathrm{l}$ TE buffer. For details see Supplementary Material.

\section{Bisulfite Conversion}

Bisulfite modification of genomic DNA was conducted with the EpiTect Plus Bisulfite Kit (Qiagen). Briefly, 1-2 $\mu \mathrm{g}$ of DNA was added to $85 \mu \mathrm{l}$ bisulfite mix and $35 \mu \mathrm{l}$ DNA Protect Buffer. Converted DNA was purified using the QIACube instrument (Qiagen).

\section{Methylation Analysis}

To determine the methylation status of six genes including FZD9, PCDH10 (protocadherin 10), SFRP2, SPARC (secreted protein acidic and rich in cysteine), UCHL1 (ubiquitin carboxyl-terminal hydrolase 1), and WIF1, we used quantitative, TaqManbased real-time PCR MethyLight adapted from Weisenberger et al. ${ }^{28}$ Primers and probes, designed specifically for bisulfite-converted fully methylated DNA are listed in Supplementary Table 1. ALU primers and probes have been used as published previously $^{20}$ and were used to normalize for the amount of input DNA. Converted CpGenome Universal Methylated DNA (Chemicon, Millipore, Billerica, MA, USA) was used as the $100 \%$ methylated control and whole genome amplified DNA (Repli-g Mini Kit; Qiagen) extracted from peripheral blood mononuclear cells of normal individuals was used as unmethylated control DNA. The $30 \mu \mathrm{l}$ MethyLight reaction mixture contained $3 \mu \mathrm{l} 10 \times$ TaqMan Buffer A (Life Technologies, Carlsbad, CA, USA), $0.1 \mu \mathrm{l}$ of AmpliTaq Gold ${ }^{\circledR}$ DNA polymerase (Life Technologies), $200 \mu \mathrm{M}$ dNTPs, $3.5 \mathrm{mM} \mathrm{MgCl}_{2}, 0.6 \mu \mathrm{M}$ of each primer, $0.2 \mu \mathrm{M}$ of each probe, and $4 \mu \mathrm{l}$ of a bisulfite-converted DNA. All assays were able to detect 1\% methylated DNA in a background of unmethylated DNA.

As DNA concentration is mostly overestimated by simple absorbance measurement and does not provide a comprehensive assessment of amplifiable DNA, we determined the amount of amplifiable DNA using $A L U$ quantitative PCR and assigned the samples to five different categories. For details see Supplementary Material and Supplementary Table 2.

On each plate, a five-point standard curve for $A L U$ (ranging from 5 to $0.0005 \mathrm{ng}$ ) was included in duplicate and used for normalizing the input DNA and converting the Ct values to mean values per copy numbers using the standard curve best-fit equation. Percentage of methylated reference was then calculated as follows: ((target mean value for sample) $/(A L U$ mean value for sample $) /($ target mean value for $100 \% \mathrm{M}) /(A L U$ mean value for $100 \% \mathrm{M})) \times 100$.

\section{Statistical Analyses}

Methylation data were generated in a blinded manner without the knowledge of any clinical data. For comparing means of two groups, the independent samples $t$-test was used. Baseline clinical data according to the methylation status were compared 
Table 1 Patient and tumor characteristics

\begin{tabular}{|c|c|c|c|}
\hline & $5-F U / L V, \mathrm{n}(\%)$ & $\begin{array}{c}\text { Surveillance, } \\
\mathrm{n}(\%)\end{array}$ & $\mathrm{P}$-value \\
\hline Number of patients $(n)$ & 71 & 72 & \\
\hline $\begin{array}{l}\text { Median age } \\
\text { (years, range) }\end{array}$ & $64.5(30.4-78.0)$ & $64.5(35.4-78.0)$ & 0.945 \\
\hline \multicolumn{4}{|l|}{ Sex } \\
\hline Female & $32(45.1)$ & $35(48.6)$ & \multirow[t]{2}{*}{0.671} \\
\hline Male & $39(54.9)$ & $37(51.4)$ & \\
\hline \multicolumn{4}{|l|}{ T category } \\
\hline T3 & $62(87.3)$ & 59 (81.9) & \multirow[t]{2}{*}{0.373} \\
\hline $\mathrm{T} 4$ & $9(12.7)$ & $13(18.1)$ & \\
\hline \multicolumn{4}{|l|}{ Grading } \\
\hline G1 and G2 & $58(81.7)$ & 59 (81.9) & \multirow[t]{2}{*}{0.969} \\
\hline G3 and G4 & $13(18.3)$ & $13(18.1)$ & \\
\hline \multicolumn{4}{|l|}{ Tumor localization } \\
\hline $\begin{array}{l}\text { Cecum/Cyathea } \\
\text { ascendens }\end{array}$ & $19(26.8)$ & $15(20.8)$ & \multirow[t]{3}{*}{0.53} \\
\hline $\begin{array}{l}\text { Flexur/Cyathea } \\
\text { transversum }\end{array}$ & $13(18.3)$ & $18(25.0)$ & \\
\hline $\begin{array}{l}\text { Sigma/Cyathea } \\
\text { descendens }\end{array}$ & $39(54.9)$ & $39(50.3)$ & \\
\hline \multicolumn{4}{|l|}{ Methylation } \\
\hline PCDH10 & $66(93.0)$ & $69(95.8)$ & \multirow[t]{3}{*}{0.989} \\
\hline$S P A R C^{\mathrm{a}}$ & $46(68.7)$ & $48(68.6)$ & \\
\hline UCHL1 & $53(74.6)$ & $51(70.8)$ & \\
\hline
\end{tabular}

${ }^{\mathrm{a}}$ The number of patients was 67 in the therapy arm and 70 in the surveillance group.

in univariate analyses using the $\chi^{2}$ test. The MannWhitney $U$-test was used for continuous variables. Survival time was defined as the period between the date of randomization and the date of death of any cause (overall survival) or the period between the date of randomization and date of first local/distant recurrence or last follow-up (disease-free survival). Patients who died before experiencing disease recurrence were censored at their date of death in the disease-free survival analysis. Survival rates were estimated by means of the Kaplan-Meier method. Differences between survival curves were analyzed by means of the log-rank test. The independent prognostic and/or predictive value of methylation status was studied with Cox models, which were adjusted for age, sex, tumor size, tumor grade, tumor localization, and treatment arm. Variables were coded as described in Table 1. These models were also applied to assess interactions between treatment and other covariates. All reported $P$-values are two-sided. For multiple comparisons, a Bonferroni-adjusted significance level of 0.007 was considered for multiple testing of seven promoters.

All analyses were performed with the use of IBM SPSS Statistics software, version 20.0

\section{Results}

\section{Selecting Candidate Genes for Methylation Analysis}

To identify aberrantly methylated promoters in colorectal cancer, we screened 46 promoters in two

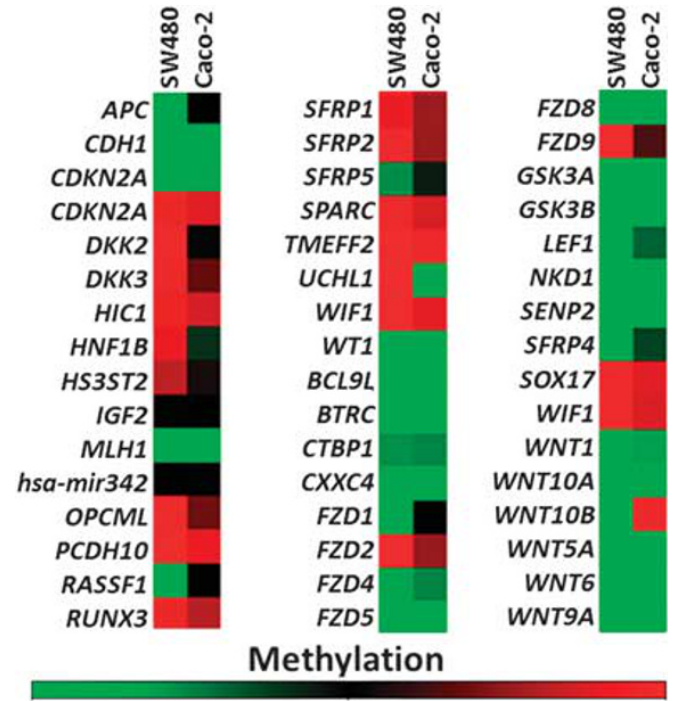

Figure 1 Heatmap of the methylation status of 46 different gene promoters of Caco-2 and SW480. Methylation analysis of 46 different promoters of the colorectal cancer cell lines, Caco-2 and SW480, using two Methyl-Profiler Assays (Qiagen) based on selective restriction digests of both methylated or unmethylated DNA, and subsequent quantitative polymerase chain reaction (PCR). Unmethylated promoters appear in green, intermediate methylation status is displayed in black, and highly methylated promoters are shown in red.

colorectal cancer cell lines (SW480 and Caco-2) using two Methyl-Profiler Assays (Qiagen). Twentyfour promoters $(52 \%)$ showed intermediate to high (50-100\%) methylation in at least one cell line (Figure 1). Sixteen promoters (DKK2, PCDH10, SFRP1, SFRP2, SPARC, UCHL1, WIF1, FZD9, SOX17, DKK3, HIC1, HFNB1, TMFFE1, and $W N T 10 B)$ were selected to investigate whether promoter methylation is associated with transcriptional silencing. Therefore, mRNA levels of three biological replicates from four different colorectal cancer cell lines (SW480, Caco2, HT29, and HCT116) treated with the demethylating agent 5-Aza were analyzed and compared with untreated control replicates. Six targets including FZD9, PCDH10, SFRP1, SPARC, UCHL1, and WIF1 showed an increase in expression of $>10$-fold in at least one analyzed cell line after 5-Aza treatment (Figure 2). To confirm that the observed increase in expression after treatment with 5-Aza was indeed associated with promoter demethylation, we measured corresponding methylation levels. The obtained percentage of methylated reference values of all six analyzed promoters decreased significantly in all four cell lines after treatment with 5-Aza $(P=0.008, P=0.010, P=0.003$, and $P=0.037$ for Caco-2, HCT116, HT29, and SW480 respectively) (Figures 2a-d). Therefore, methylation of the selected promoters was considered as potentially biologically relevant and these targets were analyzed in a cohort of 147 colorectal cancer patients. 

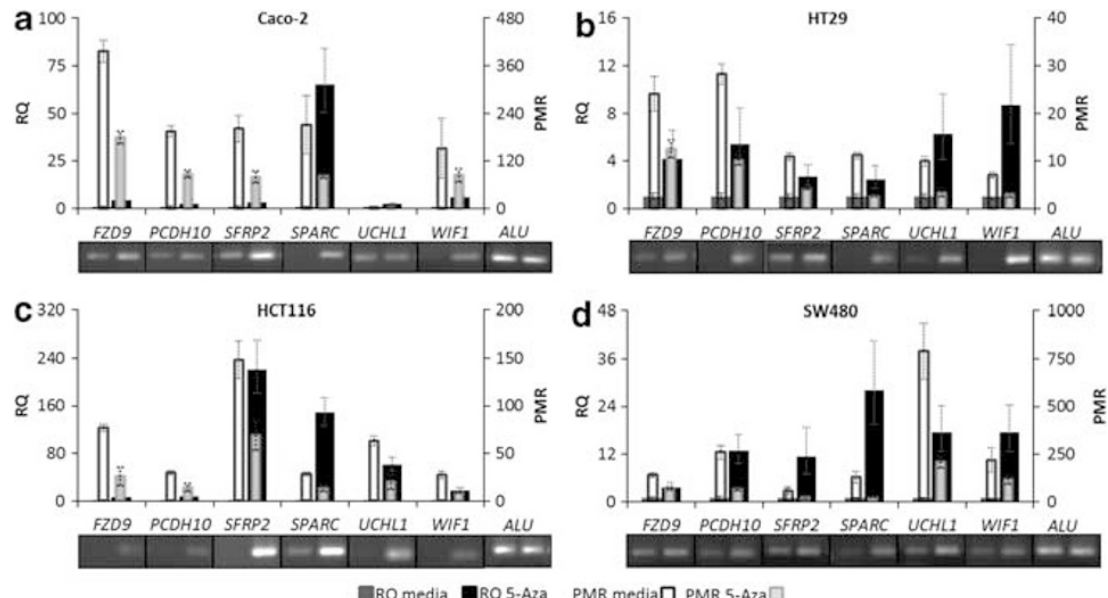

Figure 2 Expression and promoter analysis after demethylation with 5-aza-2-deoxycytidine (5-Aza) in four colorectal cancer cell lines. Caco-2, HCT116, HT29, and SW480 cells were incubated with 5-Aza (1 $\mu \mathrm{M}, 72 \mathrm{~h})$. mRNA fold change (relative quantification (RQ)) of the treated samples with respect to untreated samples was evaluated. Semiquantitative analysis is depicted under the bar charts. At the same time, promoter methylation status (percentage of methylated reference) was analyzed, to ensure that increase in expression was indeed due to promoter demethylation. Percentage of methylated reference significantly decreased in all analyzed cell lines $(P=0.008$, $P=0.010, P=0.003$, and $P=0.037$ for (a) Caco-2, (b) HCT116, (c) HT29, and (d) SW480, respectively).

\section{Patients' Characteristics}

Tumor samples $(n=147)$ were derived from patients with stage II colorectal cancer available from a previous study conducted by the Austrian Breast and Colorectal cancer Study Group. ${ }^{26}$ Four patients had to be excluded owing to poor PCR amplification in $A L U$ PCR. For SPARC six further samples had to be excluded owing to the same reason. Taken together, for a total number of 143 patients both data sets were available, methylation status of FZD9, PCDH10, SFRP2, UCHL1, and WIF1, and follow-up data. For SPARC only 137 data sets were used for statistical evaluation (Figure 3). Median follow-up of the study population was 11 years $(95 \%$ confidence interval: 10-12). The samples analyzed were from 76 male and 67 female patients, of which 71 were previously assigned to the therapy arm and 72 to the surveillance group, respectively (Figure 3). Clinical data and characteristics were well balanced between both groups (Table 1). Median age of the two groups was 64 (range 30-78) for the therapy group and 65 (range 35-78) for the surveillance group, respectively. Median overall survival was 11 years in the treatment group and 12 years in the surveillance group, respectively.

\section{Methylation Analysis of Patient Samples}

As previous studies considered a percentage of methylated reference of $\geq 4$ as biologically relevant because of a direct association with transcriptional silencing, ${ }^{29}$ we classified all samples below a percentage of methylated reference of 4 as unmethylated. All other samples were initially classified into four groups, including no, low, medium, and high methylation as illustrated in Table 2. FZD9 and PCDH1O were rarely unmethy- lated; promoter methylation was present in 96\% and $94 \%$ of all analyzable samples, respectively, whereas promoters of SFRP2, SPARC, and UCHL1 were methylated in $55 \%, 69 \%$, and $73 \%$ of all samples, respectively. Methylation of the WIF1 promoter was observed only in $33 \%$ of samples. High methylation levels were frequently found to be present in FZD9 (76\%) and PCDH10 (62\%) followed by SPARC $(44 \%)$ and UCHL1 (33\%), whereas only $11 \%$ and $4 \%$ of samples showed high methylation levels of SFPR2 and WIF1, respectively (Table 2).

Next, we used the presence or absence of promoter methylation as a continuous variable and performed a Spearman's correlation analysis (Supplementary Table 3). Except for WIF1, we observed significant positive associations among the methylation status of all analyzed promoters, indicating that combining multiple promoter methylation levels could provide greater accuracy in predicting clinical outcomes than analysis of individual markers.

A comparison of promoter methylation of all six targets between the two treatment arms showed no significant differences (Table 1). Targets that showed no prognostic relevance are not included in this table.

\section{Association of Methylation and Overall/Disease-Free Survival}

Using univariate analysis, we analyzed whether clinicopathological characteristics such as age, sex, tumor stage, grading, or localization of the tumor are associated with methylation of the six analyzed promoters. Except for one significant association between WIF1 and age $(P=0.007)$, no significant associations were observed. Next, we examined possible associations of promoter methylation status 


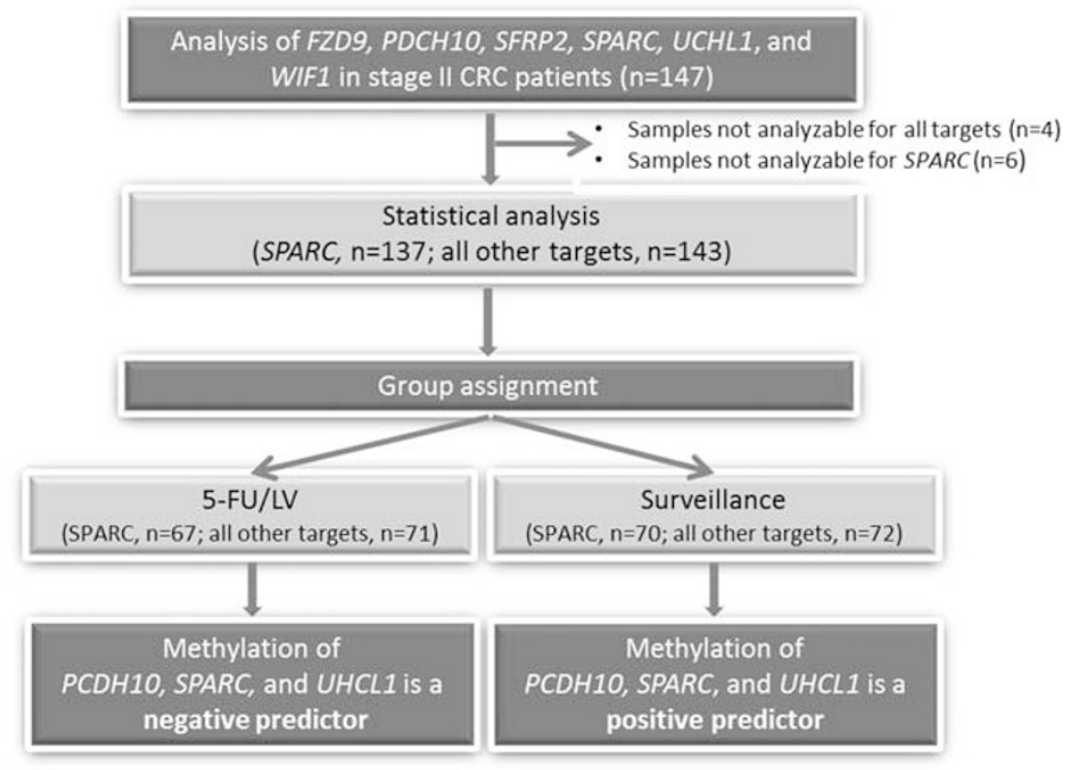

Figure 3 Flow diagram of samples enrolled in this study. A total of 147 patients were included in the study. After promoter methylation analysis of six genes and statistical analysis, a combination of three promoters turned out to be a significant predictor for therapy decisions in stage II colorectal cancer patients.

Table 2 Total percentages of methylated samples

\begin{tabular}{|c|c|c|c|c|c|c|c|}
\hline Methylation & $P M R$ & FZD9 $(\mathrm{n}=143)$ & PCDH10 $(\mathrm{n}=143)$ & SFPR2 $(\mathrm{n}=143)$ & SPARC $(\mathrm{n}=137)$ & UCHL1 (n=143) & WIF1 $(\mathrm{n}=143)$ \\
\hline Low & $>4 \leq 10$ & 4.2 & 7 & 21 & 8.8 & 6.3 & 13.3 \\
\hline Medium & $>10 \leq 50$ & 16.1 & 25.9 & 23.8 & 16.1 & 33.6 & 16.1 \\
\hline High & $>50$ & 75.5 & 61.5 & 10.5 & 43.8 & 32.9 & 3.5 \\
\hline Total methylated & $>4$ & 95.8 & 94.4 & 55.2 & 68.6 & 72.7 & 32.9 \\
\hline Unmethylated & $<4$ & 4.2 & 5.6 & 44.8 & 31.4 & 27.3 & 67.1 \\
\hline
\end{tabular}

PMR, percentage of methylated reference.

with disease-free (disease free survival) and/or overall survival (overall survival). No statistically significant differences were detected between different methylation levels (no, low, medium, high) and survival except for SPARC $(P=0.002)$ for patients with methylated promoters compared with those with no methylation. However, when patients were stratified by treatment assignment (5-fluorouracil/leucovorin or surveillance), survival analysis showed differential effects with regard to diseasefree survival and overall survival (Figure 4). Diseasefree survival tended to improve in patients of the surveillance group with methylated SPARC promoters $(P=0.036)$, whereas overall survival was significantly improved in these patients $(P=0.002)$. In contrast, methylation of SPARC was associated with shorter survival in patients who received therapy with 5-fluorouracil/leucovorin, although not statistically significant $(P=0.132$ for disease-free survival and $P=0.250$ for overall survival) (Figure 4). The same trend toward both disease-free survival and overall survival was observed for patients in the surveillance group with methylation in UCHL1 (disease-free survival $P=0.262$ and overall survival
$P=0.244$ ), whereas a nonsignificant relation between methylation and a shorter survival was observed in the chemotherapy group $(P=0.248$ for disease-free survival and $P=0.199$ for overall survival). For $P C D H 10$ similar results were obtained; however, the number of events was too small to obtain accurate $P$-values. Combined evaluation of all three methylation markers showed an association between methylation and a shorter survival $(P=0.069$ for disease-free survival and $P=0.139$ for overall survival) in the chemotherapy arm. However, patients in the surveillance arm had a significantly prolonged overall survival $(P=0.031$ for disease-free survival and $P=0.003$ for overall survival) (Figure 4).

To determine a possible interaction between the combined methylation markers and treatment, an interaction term, the product of combined methylation markers and treatment, was incorporated into the Cox models. These analyses revealed a strong interaction between combined methylation markers and treatment (test for interaction, $P=0.006$ for disease-free survival and $P=0.018$ for overall survival). 

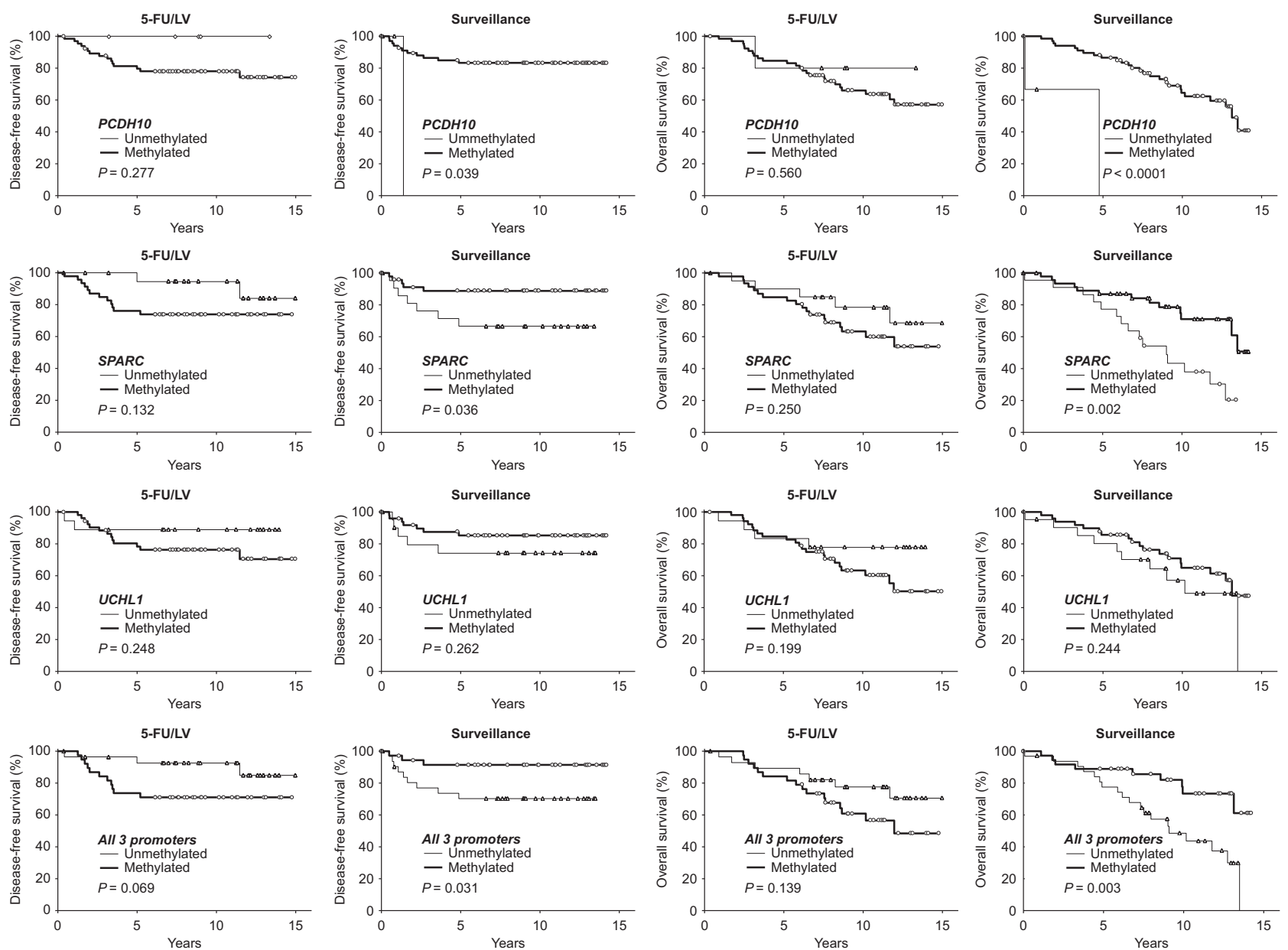

Figure 4 Kaplan-Meier estimates of disease-free and overall survival in colorectal cancer patients treated with (5-fluorouracil/ leucovorin) or without (surveillance) chemotherapy according to the presence or absence of methylated promoters. Data are reported for a panel of three promoters consisting of PCDH10 (protocadherin 10), SPARC (secreted protein acidic and rich in cysteine), and UCHL1 (ubiquitin carboxyl-terminal hydrolase 1). Methylation of one (with the exception of UCHL1) or a combination of all three promoters was partly significantly associated with a better outcome in the surveillance group. In contrast, promoter methylation decreased survival rates in patients who received chemotherapy, although not statistically significant. $P$-values are based on the log-rank test. Owing to multiple testing Bonferroni-adjusted $P$-values $<0.007$ were considered to be statistically significant. *NA, not analyzable-the number of events was too small to obtain accurate $P$-values.

\section{Discussion}

Our study indicates that promoter methylation status of three genes including PCDH10, SPARC, and UCHL1 may help to identify patients who might benefit from chemotherapy, and consequently facilitate treatment decisions for stage II colorectal cancer patients. All analyzed tumor samples were available from a previous study conducted by the Austrian Breast and Colorectal cancer Study Group, where the efficacy of adjuvant chemotherapy with 5 -fluorouracil and leucovorin in stage II colon cancer was investigated. ${ }^{26}$ In the course of this study, patients were randomly stratified to either adjuvant chemotherapy with 5-fluorouracil/ leucovorin or surveillance only. However, no statistically significant improvement on diseasefree survival and/or overall survival could be achieved by the application of chemotherapy in this study. This highlights the need for additional prognostic markers that may prospectively identify patients who are likely to be cured with chemotherapy versus those patients who have a better prognosis after surgery only and would not benefit from chemotherapy.

Our study revealed that promoter methylation of UCHL1 and SPARC was correlated with a better clinical outcome in the surveillance group compared with patients without methylation at these promoters (Figure 4). In contrast, patients of the chemotherapy group, who were methylated at UCHL1 and SPARC promoter sites, seemed to have poorer overall survival and disease-free survival. A similar trend was observed for methylation of PCDH10 (Figure 4). A combined analysis of all three promoters was in concordance with the analysis of individual markers, with the trend for better survival or relapse time being even improved. This was also reflected in the Spearman's correlation analysis, where the methylation status of almost all 
analyzed promoters was significantly correlated, indicating that a combined analysis of more promoter regions could provide greater clinical reliability.

The involvement of PCDH10, SPARC, and UCHL1 in tumor formation and progression is well documented in the literature. PCDH10 is a member of the cadherin superfamily, which is known to be a cellcell adhesion molecule. In the context of tumorigenesis, PCDH10 is found to have a role in growth control, tumor cell invasion, and metastasis, ${ }^{30}$ and has recently been proposed to be a tumor suppressor gene. $^{31}$ PCDH10 methylation was frequently reported in several solid tumors and was closely associated with malignancy and negative outcome. $^{32,33} \mathrm{In}$ a recent study from $\mathrm{Yu}$ et $\mathrm{al}^{33}$ and Lin et al, ${ }^{34}$ PCDH10 promoter methylation was detected in $85 \%$ of primary colorectal tumors and all analyzed colorectal cancer cell lines. This is in concordance with our data where PCDH10 methylation was found in more than $90 \%$ of patients. However, in our study, poor prognosis due to methylation, as reported previously, ${ }^{33,34}$ was observed only for the chemotherapy group, although not being significant. For the surveillance group methylation of $\mathrm{PCDH} 10$ promoter showed a significantly better survival and relapse rate. In this context, it should be noted that only very few patients showed no PCDH1O methylation and the number of events was too small for reliable calculations of error probabilities; therefore, these data should be considered as preliminary and further investigations have to be conducted.

SPARC is described to be involved in endothelial permeability, regulation of endothelial barrier function, and cell migration and differentiation. Therefore, SPARC is predestinated to have a role in tumor angiogenesis, proliferation, and cell migration. However, the role of SPARC as an oncogene or a tumor suppressor gene is still not fully elucidated. SPARC has been reported to act as a protumorigenic and antitumorigenic protein in different tumor entities. ${ }^{35}$ For colorectal cancer also conflicting results have been reported. Expression of SPARC was shown to be significantly higher in tumor tissue than in normal colon or matched non-malignant counterparts. ${ }^{36,37}$ Therefore, SPARC was suggested as a potentially useful target candidate for cancer immunotherapy. ${ }^{36}$ Yoshimura et $a l^{38}$ reported a higher recurrence risk for colorectal cancer by an upregulated SPARC expression in adjacent tumor tissue. These data are consistent with our findings, where a decreased level of SPARC due to promoter methylation was associated with a better clinical outcome. This is also supported from animal models were SPARC deficiency strongly suppressed adenoma formation in the intestine in Sparc knockout mice intercrossed with Apc(Min/ + ) mice. ${ }^{39}$

In contrast, a significant association of $S P A R C$ methylation and poorer prognosis was reported by Yang et al. ${ }^{40}$ Although the authors analyzed a large cohort of patients, they did not differentiate between tumor stages nor did they consider stratification by chemotherapy. In our study, SPARC promoter methylation tended to be associated with shortened overall survival and disease-free survival, only in patients who received chemotherapy. These results are supported by the fact that decreased SPARC expression has been observed in therapy-refractory tumors compared with those sensitive to chemotherapy, ${ }^{41}$ suggesting that upregulation of SPARC expression might enhance chemosensitivity. ${ }^{42}$ Hence, the slightly worse outcome for patients with SPARC methylation may be a result of decreased chemotherapy sensitivity in the absence of SPARC due to epigenetic silencing. ${ }^{43}$

Similar to SPARC, the role of UCHL1 in tumor development is still controversially discussed. ${ }^{4}$ The gene product of UCHL1 is known to catalyze deubiquitination of proteins and is typically expressed in neurons. Mutations in UCHL1 are closely linked to the development of Parkinson disease, and are also found in neuronal tumors. However, recent studies indicate the involvement in other human non-neural cancers, such as breast, colorectal, and pancreatic cancer. ${ }^{44}$ Data from several in vitro studies and mouse models suggest that UCHL1 acts as an oncogene in cancer pathogenesis. ${ }^{45-47}$ Furthermore, high expression of UCHL1 in cancerassociated fibroblasts was shown as an independent, negative prognostic factor for overall and recurrence-free survival. ${ }^{45}$ Albo et $a l^{48}$ reported that neurogenesis-in which UCHL1 is a key player-in colorectal cancer is involved in tumor progression and associated with poor outcome. In 2008, Mizukami et $a l^{49}$ proposed that hypomethylation resulting in re-expression of UCHL1 was significantly associated with lymph node metastasis in colorectal cancer. These data strongly support our findings where patients under surveillance tended to have a better outcome when UCHL1 promoter was methylated compared with patients who showed no methylation and therefore most likely had increased expression levels of UCHL1.

On the contrary, UCHL1 was also reported to act as a tumor suppressor ${ }^{50}$ and promoter methylation has been suggested as an independent prognostic factors in different cancers. ${ }^{51}$ In our data set, UCHL1 methylation was associated with a shortened overall survival and disease-free survival only in patients receiving chemotherapy. Whether the opposing results from the therapy group can be explained by decreased chemotherapy sensitivity, similar to $S P A R C$, remains to be elucidated.

Although further studies are needed to clarify the exact mechanisms of PCDH10, SPARC, and UCHL1 in tumorigenesis and progression, and their interaction with the chemotherapy regimen, our data suggest that analysis of promoter methylation in tumor tissue may provide a suitable tool for determination of prognosis of stage II colorectal cancer patients. More importantly, methylation of PCDH10, SPARC, and UCHL1 may predict response 
to the adjuvant treatment. Therefore, our results could help to stratify early-stage colorectal cancer patients into a high-risk group promoter methylation of selected genes that would benefit from adjuvant chemotherapy.

To date, adjuvant chemotherapy for stage II colorectal cancer patients is still controversially discussed. Even if chemotherapeutic treatment has improved and the risk of toxicities has become more calculable, the guidelines from the American Society of Clinical Oncology (ASCO) from 2004 do not recommend a general treatment for patients in stage II. ${ }^{52}$

In conclusion, we could demonstrate that analyses of methylation in our selected genes might be used to determine patients with worse prognosis, as demonstrated in untreated patients, and that selection of these patients for adjuvant treatment may predict the benefit of chemotherapy. However, these arguments are based on a retrospective study with a relatively low number of cases. Furthermore, we cannot exclude confounding effects like BRAF mutations or resistance to 5-fluorouracil by microsatellite instability, which were previously shown to influence survival in colorectal cancer patients. ${ }^{53,54}$ However, owing to the lack of material and bad DNA quality, it was not possible to assess additional genetic markers in our study cohort. In conclusion, the identification and validation of new biomarker is challenging owing to several reasons (for a detailed review see Febbo et $a 1^{55}$ ); therefore, our results should be evaluated together with other prognostic factors such as BRAF mutations prospectively in a larger clinical trial.

\section{Acknowledgments}

This research was supported by funds of the Oesterreichische Nationalbank (Anniversary Fund, Project Number: 13425 to EH).

\section{Disclosure/conflict of interest}

The authors declare no conflict of interest.

\section{References}

1 Soreide K, Berg M, Skudal BS, et al. Advances in the understanding and treatment of colorectal cancer. Discov Med 2011;12:393-404.

2 Leong SP, Cady B, Jablons DM, et al. Patterns of metastasis in human solid cancers. Cancer Treat Res 2007;135:209-221.

3 Andre T, Afchain P, Barrier A, et al. Current status of adjuvant therapy for colon cancer. Gastrointest Cancer Res 2007;1:90-97.

4 Jonker DJ, Spithoff K, Maroun J. Gastrointestinal Cancer Disease Site Group of Cancer Care Ontario's Program in Evidence-based C. Adjuvant systemic chemotherapy for Stage II and III colon cancer after complete resection: an updated practice guideline. Clin Oncol (R Coll Radiol) 2011;23:314-322.

5 Berg M, Soreide K. Genetic and epigenetic traits as biomarkers in colorectal cancer. Int J Mol Sci 2011;12: 9426-9439.

6 Popat S, Hubner R, Houlston RS. Systematic review of microsatellite instability and colorectal cancer prognosis. J Clin Oncol 2005;23:609-618.

7 Ogino S, Nosho K, Kirkner GJ, et al. CpG island methylator phenotype, microsatellite instability, BRAF mutation and clinical outcome in colon cancer. Gut 2009;58:90-96.

8 Chen Y, Yi C, Liu L, et al. Thymidylate synthase expression and prognosis in colorectal cancer: a metaanalysis of colorectal cancer survival data. Int J Biol Markers 2012;27:e203-e211.

9 Gray RG, Quirke P, Handley K, et al. Validation study of a quantitative multigene reverse transcriptase-polymerase chain reaction assay for assessment of recurrence risk in patients with stage II colon cancer. J Clin Oncol 2011;29:4611-4619.

10 Wang Y, Jatkoe T, Zhang Y, et al. Gene expression profiles and molecular markers to predict recurrence of Dukes’ B colon cancer. J Clin Oncol 2004;22:1564-1571.

11 Myklebust MP, Li Z, Tran TH, et al. Expression of cyclin D1a and D1b as predictive factors for treatment response in colorectal cancer. Br J Cancer 2012; 107:1684-1691.

12 Andreyev HJ, Norman AR, Cunningham D, et al. Kirsten ras mutations in patients with colorectal cancer: the 'RASCAL II' study. Br J Cancer 2001;85:692-696.

13 Rawson JB, Bapat B. Epigenetic biomarkers in colorectal cancer diagnostics. Expert Rev Mol Diagn 2012;12:499-509.

14 Harder J, Engelstaedter V, Usadel H, et al. CpG-island methylation of the ER promoter in colorectal cancer: analysis of micrometastases in lymph nodes from UICC stage I and II patients. Br J Cancer 2009;100:360-365.

15 Kawakami K, Ruszkiewicz A, Bennett G, et al. DNA hypermethylation in the normal colonic mucosa of patients with colorectal cancer. Br J Cancer 2006;94: 593-598.

16 Miranda E, Destro A, Malesci A, et al. Genetic and epigenetic changes in primary metastatic and nonmetastatic colorectal cancer. Br J Cancer 2006;95:1101-1107.

17 Zhang YQ, Pilyugin M, Kuester D, et al. Expression of oncogenic BARD1 isoforms affects colon cancer progression and correlates with clinical outcome. Br J Cancer 2012;107:675-683.

18 Lao VV, Grady WM. Epigenetics and colorectal cancer. Nat Rev Gastroenterol Hepatol 2011;8:686-700.

19 Ogino S, Lochhead P, Chan AT, et al. Molecular pathological epidemiology of epigenetics: emerging integrative science to analyze environment, host, and disease. Mod Pathol 2013;26:465-484.

20 Toyota M, Ahuja N, Ohe-Toyota M, et al. CpG island methylator phenotype in colorectal cancer. Proc Natl Acad Sci USA 1999;96:8681-8686.

21 Van Rijnsoever M, Elsaleh H, Joseph D, et al. CpG island methylator phenotype is an independent predictor of survival benefit from 5-fluorouracil in stage III colorectal cancer. Clin Cancer Res 2003;9:2898-2903.

22 Shen L, Catalano PJ, Benson AB III, et al. Association between DNA methylation and shortened survival in patients with advanced colorectal cancer treated with 5-fluorouracil based chemotherapy. Clin Cancer Res 2007;13:6093-6098. 
23 Krakowczyk L, Strzelczyk JK, Adamek B, et al. Methylation of the MGMT and p16 genes in sporadic colorectal carcinoma and corresponding normal colonic mucosa. Med Sci Monit 2008;14:BR219-BR225.

24 Nilsson TK, Lof-Ohlin ZM, Sun XF. DNA methylation of the p14ARF, RASSF1A and APC1A genes as an independent prognostic factor in colorectal cancer patients. Int J Oncol 2013;42:127-133.

25 Grunau C, Brun ME, Rivals I, et al. BAGE hypomethylation, a new epigenetic biomarker for colon cancer detection. Cancer Epidemiol Biomarkers Prev 2008; 17:1374-1379.

26 Schippinger W, Samonigg H, Schaberl-Moser R, et al. A prospective randomised phase III trial of adjuvant chemotherapy with 5-fluorouracil and leucovorin in patients with stage II colon cancer. Br J Cancer 2007;97: 1021-1027.

27 Balic M, Pichler M, Strutz J, et al. High quality assessment of DNA methylation in archival tissues from colorectal cancer patients using quantitative high-resolution melting analysis. J Mol Diagn 2009;11:102-108.

28 Weisenberger DJ, Siegmund KD, Campan M, et al. CpG island methylator phenotype underlies sporadic microsatellite instability and is tightly associated with BRAF mutation in colorectal cancer. Nat Genet 2006;38:787-793.

29 Ogino S, Kawasaki T, Brahmandam M, et al. Precision and performance characteristics of bisulfite conversion and real-time PCR (MethyLight) for quantitative DNA methylation analysis. J Mol Diagn 2006;8:209-217.

30 Jeanes A, Gottardi CJ, Yap AS. Cadherins and cancer: how does cadherin dysfunction promote tumor progression? Oncogene 2008;27:6920-6929.

31 Ying J, Li H, Seng TJ, et al. Functional epigenetics identifies a protocadherin PCDH10 as a candidate tumor suppressor for nasopharyngeal, esophageal and multiple other carcinomas with frequent methylation. Oncogene 2006;25:1070-1080.

32 Lin YL, Li ZG, Guan TY. The clinical significance of PCDH10 promoter methylation in patients with bladder transitional cell carcinoma. Urol Int 2012;90:219-224.

$33 \mathrm{Yu}$ J, Cheng YY, Tao Q, et al. Methylation of protocadherin 10, a novel tumor suppressor, is associated with poor prognosis in patients with gastric cancer. Gastroenterology 2009;136:e1.

34 Lin YL, Li ZG, He ZK, et al. Clinical and prognostic significance of protocadherin-10 (PCDH10) promoter methylation in bladder cancer. J Int Med Res 2012; 40:2117-2123.

35 Podhajcer OL, Benedetti L, Girotti MR, et al. The role of the matricellular protein SPARC in the dynamic interaction between the tumor and the host. Cancer Metast Rev 2008;27:523-537.

36 Inoue M, Senju S, Hirata S, et al. Identification of SPARC as a candidate target antigen for immunotherapy of various cancers. Int J Cancer 2010;127:1393-1403.

37 Kaiser S, Park YK, Franklin JL, et al. Transcriptional recapitulation and subversion of embryonic colon development by mouse colon tumor models and human colon cancer. Genome Biol 2007;8:R131.

38 Yoshimura T, Nagahara M, Kuo C, et al. Lymphovascular invasion of colorectal cancer is correlated to SPARC expression in the tumor stromal microenvironment. Epigenetics 2011;6:1001-1011.
39 Sansom OJ, Mansergh FC, Evans MJ, et al. Deficiency of SPARC suppresses intestinal tumorigenesis in APCMin/ + mice. Gut 2007;56:1410-1414.

40 Yang E, Kang HJ, Koh KH, et al. Frequent inactivation of SPARC by promoter hypermethylation in colon cancers. Int J Cancer 2007;121:567-575.

41 Tai IT, Dai M, Owen DA, et al. Genome-wide expression analysis of therapy-resistant tumors reveals SPARC as a novel target for cancer therapy. J Clin Invest 2005;115:1492-1502.

42 Cheetham S, Tang MJ, Mesak F, et al. SPARC promoter hypermethylation in colorectal cancers can be reversed by 5 -Aza-2'deoxycytidine to increase SPARC expression and improve therapy response. Br J Cancer 2008;98:1810-1819.

43 Chan JM, Ho SH, Tai IT. Secreted protein acidic and rich in cysteine-induced cellular senescence in colorectal cancers in response to irinotecan is mediated by P53. Carcinogenesis 2010;31:812-819.

44 Hurst-Kennedy J, Chin LS, Li L. Ubiquitin C-terminal hydrolase 11 in tumorigenesis. Biochem Res Int 2012; 2012:123706.

45 Akishima-Fukasawa Y, Ino Y, Nakanishi Y, et al. Significance of PGP9.5 expression in cancer-associated fibroblasts for prognosis of colorectal carcinoma. Am J Clin Pathol 2010;134:71-79.

46 Kim HJ, Kim YM, Lim S, et al. Ubiquitin C-terminal hydrolase-L1 is a key regulator of tumor cell invasion and metastasis. Oncogene 2009;28:117-127.

47 Ma Y, Zhao M, Zhong J, et al. Proteomic profiling of proteins associated with lymph node metastasis in colorectal cancer. J Cell Biochem 2010;110:1512-1519.

48 Albo D, Akay CL, Marshall CL, et al. Neurogenesis in colorectal cancer is a marker of aggressive tumor behavior and poor outcomes. Cancer 2011;117:4834-4845.

49 Mizukami H, Shirahata A, Goto T, et al. PGP9.5 methylation as a marker for metastatic colorectal cancer. Anticancer Res 2008;28:2697-2700.

$50 \mathrm{Li} \mathrm{L}$, Tao Q, Jin H, et al. The tumor suppressor UCHL1 forms a complex with p53/MDM2/ARF to promote p53 signaling and is frequently silenced in nasopharyngeal carcinoma. Clin Cancer Res 2010;16:2949-2958.

$51 \mathrm{Kim}$ JH, Jung EJ, Lee HS, et al. Comparative analysis of DNA methylation between primary and metastatic gastric carcinoma. Oncol Rep 2009;21:1251-1259.

52 Benson AB III, Schrag D, Somerfield MR, et al. American Society of Clinical Oncology recommendations on adjuvant chemotherapy for stage II colon cancer. J Clin Oncol 2004;22:3408-3419.

53 Funkhouser WK Jr., Lubin IM, Monzon FA, et al. Relevance, pathogenesis, and testing algorithm for mismatch repair-defective colorectal carcinomas: a report of the association for molecular pathology. J Mol Diagn 2012;14:91-103.

54 Gavin PG, Colangelo LH, Fumagalli D, et al. Mutation profiling and microsatellite instability in stage II and III colon cancer: an assessment of their prognostic and oxaliplatin predictive value. Clin Cancer Res 2012;18: 6531-6541.

55 Febbo PG, Ladanyi M, Aldape KD, et al. NCCN Task Force report: evaluating the clinical utility of tumor markers in oncology. J Natl Compr Canc Netw 2011;9(Suppl 5):S1-32.

Supplementary Information accompanies the paper on Modern Pathology website (http://www.nature.com/ modpathol) 